\title{
Man with New-Onset Jaundice and Severe Cholestasis
}

Aarati Malliah, $M D$

\section{Case Presentation}

The patient is a 69 year-old male with new onset jaundice of two weeks and a 6 month history of progressive hyperbilirubinemia with cholestasis who was admitted for evaluation. Physical examination revealed a well developed and well nourished, jaundiced male with scleral icterus, a II/VI systolic murmur at apex, mild abdominal discomfort over epigastrum on palpation without discernable hepatomegaly, normal distal pulses, no lower extremity edema, and no stigmata of chronic liver disease. Laboratory investigation revealed a total bilirubin of $19.3 \mathrm{mg} / \mathrm{dL}$, alkaline phosphatase $693 \mathrm{U} / \mathrm{L}$, and AST and ALT of $140 \mathrm{U} / \mathrm{L}$ and $117 \mathrm{U} / \mathrm{L}$, respectively. CT scan of the abdomen and pelvis showed multiple foci of arterial enhancement, periportal edema without biliary dilatation, concerning for acute fulminant hepatitis. The patient had a dramatic and rapid decline the day following admission, developing cardiac arrhythmia and hypotension and subsequently died despite resuscitative efforts.

Autopsy revealed amyloidosis AL, kappa light chain type involving the liver (Figures 1 and 2), heart (Figures 3 and 4), lungs and bone

\section{Discussion}

Although hepatic involvement is a common manifestation of primary amyloidosis, systemic amyloid presenting with jaundice and severe cholestasis is rare and portends a poor prognosis. Amyloidosis manifesting as cholestatic liver disease has the shortest mean survival of any presentation of the disorder, approximately 3 months from the identification of jaundice; only $25 \%$ of patients live longer than 6 months. While the most common cause of death in amyloid is due to congestive cardiomyopathy or sudden death due to VF or asystole, the presence of heart failure suggests a six month clinical predictor of survival. Patients with AL often suffer from rapid-onset heart failure.

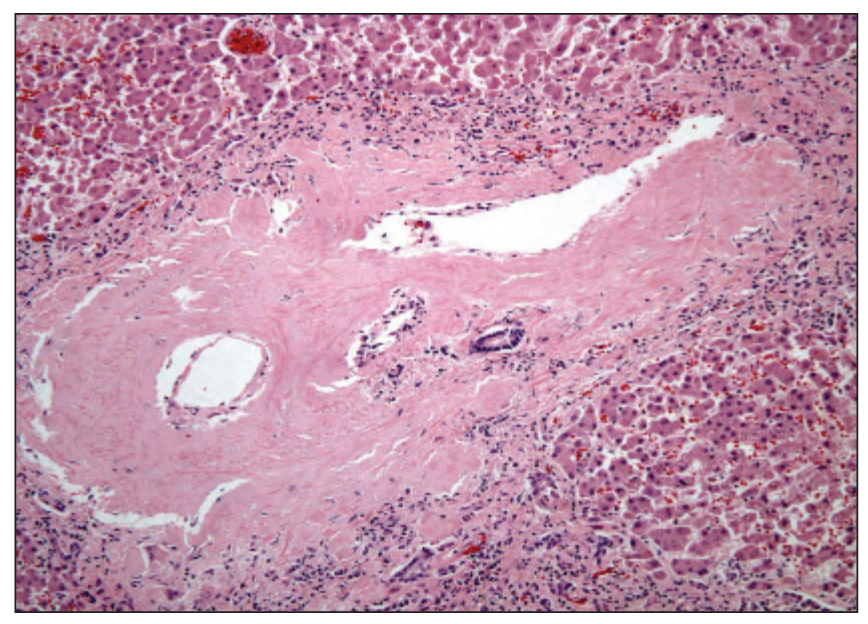

Figure 1. Liver portal triad

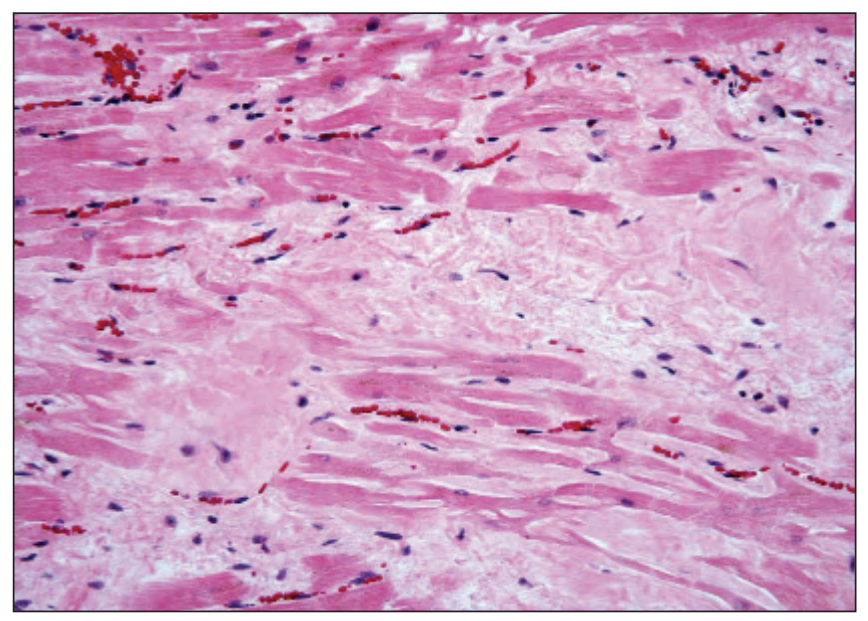

Figure 2. Liver portal triad with Congo stain

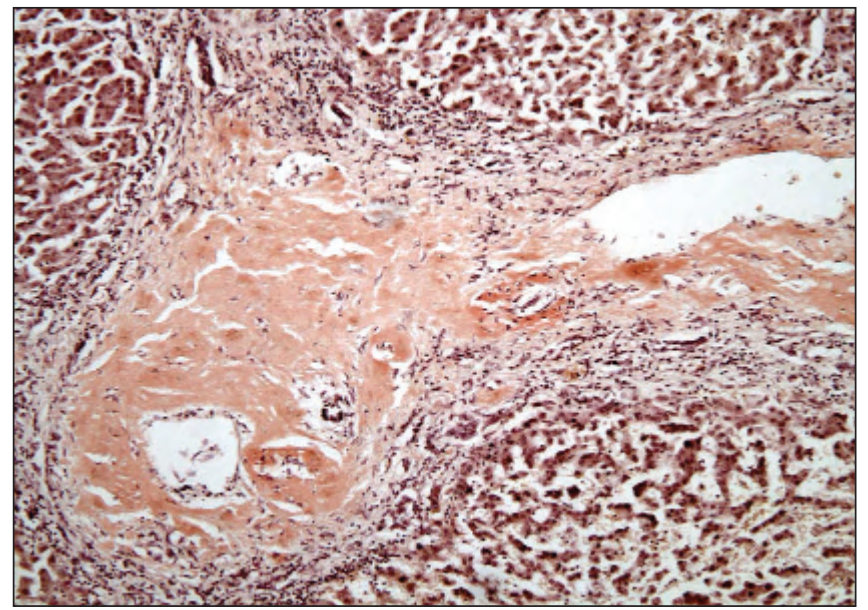

Figure 3. Left ventricle

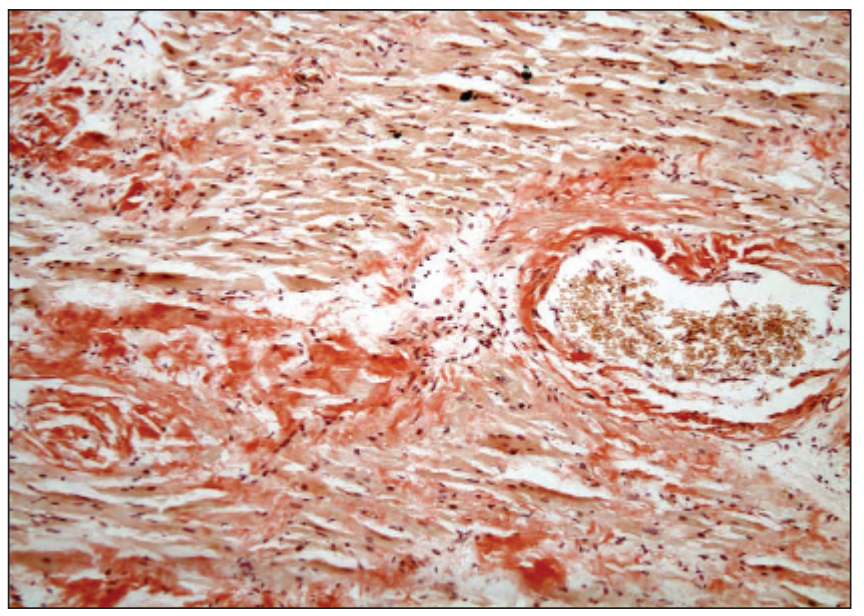

Figure 4. Left ventricle with Congo stain 